\title{
A new alternating copolymerized derivative as a cold flow improver for diesel fuel ${ }^{*}$
}

\author{
ZHANG Haikuan ${ }^{1,2}$, LIU Hongyan ${ }^{1}$ and WANG Shujun ${ }^{1 * *}$ \\ ${ }^{1}$ State Key Laboratory of Heavy Oil Processing, China University of Petroleum, Beijing 102249, China \\ ${ }^{2}$ China National Oil \& Gas Exploration and Development Corporation, China National Petroleum Corporation, Beijing 100034, \\ China
}

\begin{abstract}
Synthesis of a cold flow improver (MAVA-a) for diesel fuel and its effect on solidifying point (SP) and cold filter plugging point (CFPP) of diesel fuels were investigated. The cold flow improver was prepared by using maleic anhydride (MA) and vinyl acetate (VA) as raw materials, toluene as solvent, dibenzoyl peroxide (BPO) as initiator, through alternating polymerization under nitrogen to obtain a binary-polymer and then through aminolysis by using a higher carbon amine as aminating agent at a temperature of $80^{\circ} \mathrm{C}$. A cold flow improver was designed and prepared for No. 0 diesel fuel from Zhang Jia-Gang Petrochemical Company according to the contents of n-paraffin and its carbon number distribution in the No. 0 diesel fuel. It was also used together with two kinds of ethene-vinyl acetate copolymer improvers (EVA) separately. The test result showed that the CFPP of the No. 0 diesel fuel could be lowered by $3-5^{\circ} \mathrm{C}$ when the improver MAVA-a was used. The CFPP was lowered by $8^{\circ} \mathrm{C}$ when the improver MAVA-a was used together with EVA-2.
\end{abstract}

Key words: Cold flow improver, solidifying point, cold filter plugging point, copolymer, amination

\section{Introduction}

A small amount of additive can effectively disperse the wax of distillate fuels (Semwal and Varshney, 1995; Yang and Chang, 1997). This kind of additive is referred to a cold flow improver (CFI). It is often used in combination with conventional flow improvers to further modify the size and shape of treated wax crystals. The efficiency of CFI is related not only to its structural constitution but also to many factors of diesel fuel, such as the type of crude oil, processing technology, blending methods and composition of distillation cuts.

In recent years, increasing attention has been paid to maleic anhydride (MA) and vinyl acetate (VA) copolymer (MAVA), which is used to improve diesel fuel property (Zhang et al, 1995; Song et al, 2005), decrease wax crystal size and inhibit the formation of crystal lattice. In our work, the distribution of carbon number of n-paraffin and their contents in diesel fuel were analyzed, and a MAVA alternating copolymer and its amination derivative (MAVA-a) were prepared and characterized, and the performance of the cold flow improver (MAVA-a) was evaluated with No. 0 diesel oil of Zhang Jia-Gang Petrochemical Company.

\section{Experimental}

\footnotetext{
*Supported by the Basic Research Program of the State Key Laboratory of Heavy Oil Processing (200310), China University of Petroleum, Beijing, China

**Corresponding author. email: bjwsjbj@sina.com

Received December 28, 2007
}

\subsection{Chemicals and instruments}

Copolymer MAVA-a was synthesized and purified in the laboratory. A stearamide (purity $\geq 95 \%$ ), which is of technical grade, was provided by Shandong Boxing Chemical Company. Vinyl acetate (VA, purity $\geq 99 \%$ ) was obtained from the Fine Chemistry Trial Plant of Nankai University. Maleic anhydride (MA, purity $\geq 99.5 \%$ ) was obtained from the Beijing Chemical Reagent Company.

The multi-functional tester (SWXK 402D) for petroleum products at a low temperature, which was used for determining the cold filter plugging point depression ( $\triangle$ CFPP) in our work, was made by Shanghai Pengpu Refrigeration Company, Ltd. China; The Spectrum Analyzer (MAGNA-IR 560) was made by Nicolet company, Ltd., U.S.A; The gas chromatography (ABGC8000) was made by YOUNG LIN INSTRUMENT company, Ltd., Korea.

\subsection{Fuel composition}

The distribution of n-paraffin carbon number and the average carbon number of the No.0 diesel oil from Zhang Jia-Gang Petrochemical Company were determined by a gas chromatograph (ABGC8000) with AN-5 capillary column $(30 \mathrm{~m} \times 0.25 \mathrm{~mm} \times 0.25 \mu \mathrm{m})$ and the results are shown in Fig. 3 .

\subsection{Synthesis}

2.3.1 Copolymerization of vinyl acetate and maleic anhydride

Maleic anhydride (MA) and toluene solvent were added to a $250-\mathrm{mL}$ four-neck round flask fitted with a nitrogencontrolled inlet valve, a condenser, a temperature controller and a mechanical stirrer. The flask was flushed with nitrogen 
gas, and then the reaction mixture was gradually heated. The flow of nitrogen was continuous and vigorous stirring was applied simultaneously. When the temperature of reaction mixture reached $80^{\circ} \mathrm{C}$, vinyl acetate (VA) and benzoyl peroxide dispersed in $10-\mathrm{mL}$ of toluene were added to the reaction mixture gradually. The reaction temperature was kept at $80^{\circ} \mathrm{C}$ for several hours.

The reaction of polymerization is as follows:

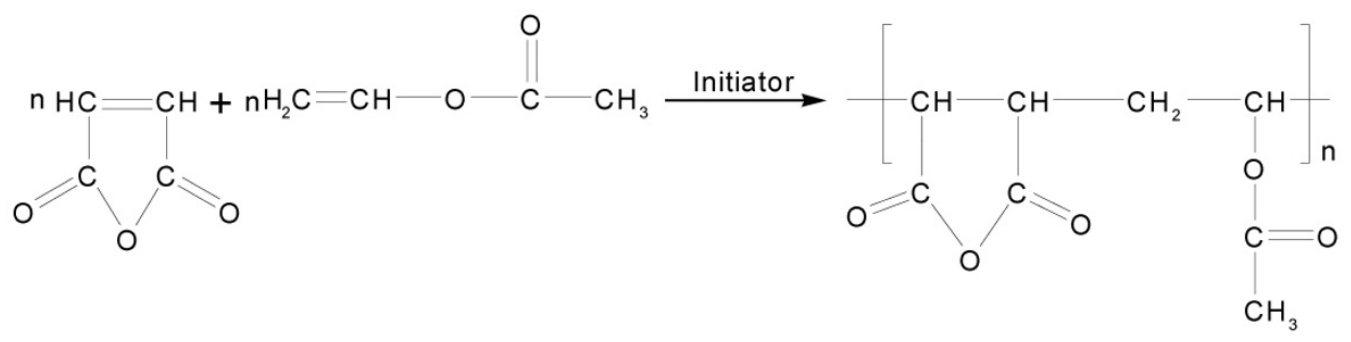

\subsubsection{Amidation of copolymers}
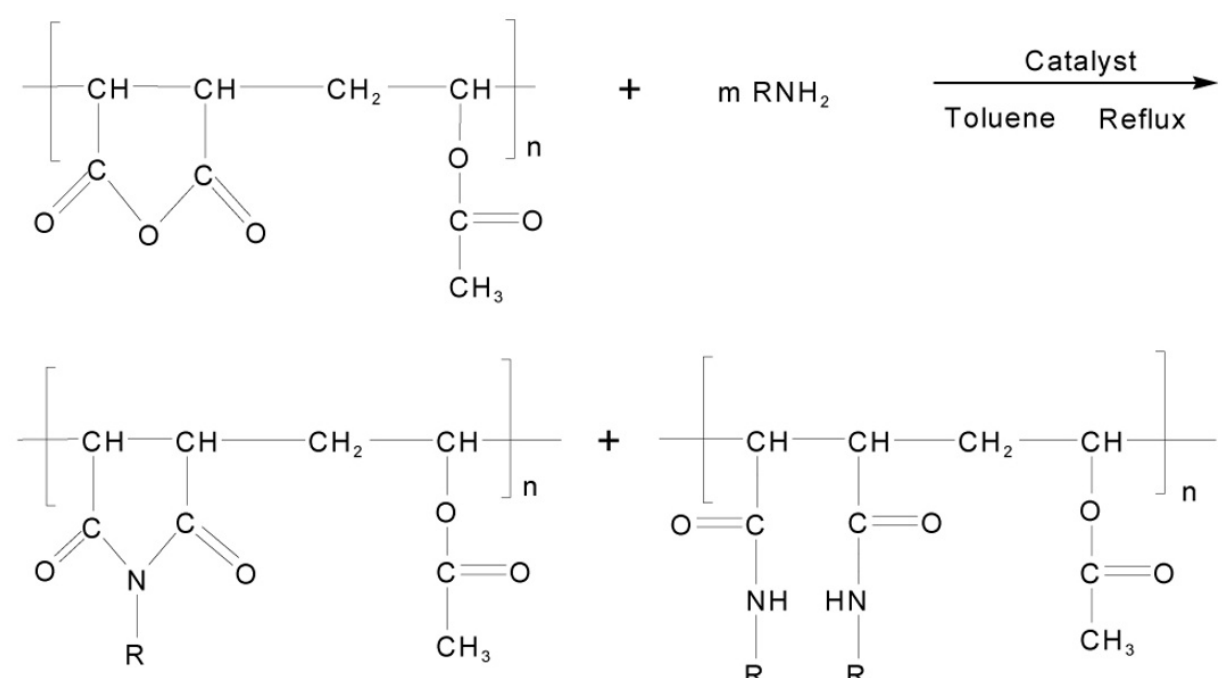

The reaction of amidation is as follows:

The copolymers MAVA were partially amidated with stearamide in a molar ratio of 1:2 (n-M: n-a, "M" is the same to MA, "a" is the amido group of the stearamide), respectively, on the basis of maleic anhydride in the copolymer. The amidation reaction took place at the reflux temperature under vigorous stirring in toluene solvent until the reaction product water was azeotropically collected in the trap. The amidated copolymers were purified under reduced pressure, and then precipitated in an excess volume of ethanol, filtered and dried in vacuum at $50{ }^{\circ} \mathrm{C}$ for several hours at last.

\section{Results and discussion}

\subsection{Characterization of chemical structures of copolymers}

The chemical structures of copolymer MAVA and amidated copolymer MAVA-a were studied by means of infrared spectrometry in $\mathrm{KBr}$ disks. The scanning range was from 4,000 to $400 \mathrm{~cm}^{-1}$, the differentiating rate was $0.35 \mathrm{~cm}^{-1}$, and the signal-to-noise ratio was 30,000:1. By comparing Fig. 1 with Fig. 2, it can be clearly seen that the stretching peaks

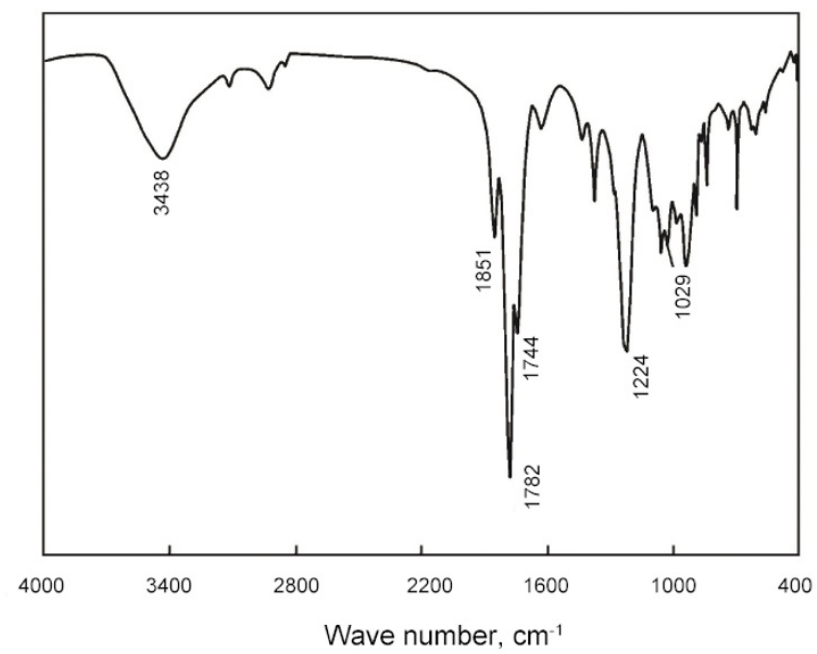

Fig. 1 IR spectra of copolymer MAVA

of the characteristic $\mathrm{C}=\mathrm{O}$ of maleic anhydride are at 1,851 and $1,782 \mathrm{~cm}^{-1}$ and the absorption peak of the characteristic $\mathrm{C}=\mathrm{O}$ of saturated ester is at $1,744 \mathrm{~cm}^{-1}$. This result indicated that the copolymer was obtained.

It can be seen in Fig. 2 that the absorption peaks of characteristic $\mathrm{CH}_{3}$ and $\mathrm{CH}_{2}$ of long- or short-chain alkyl are 


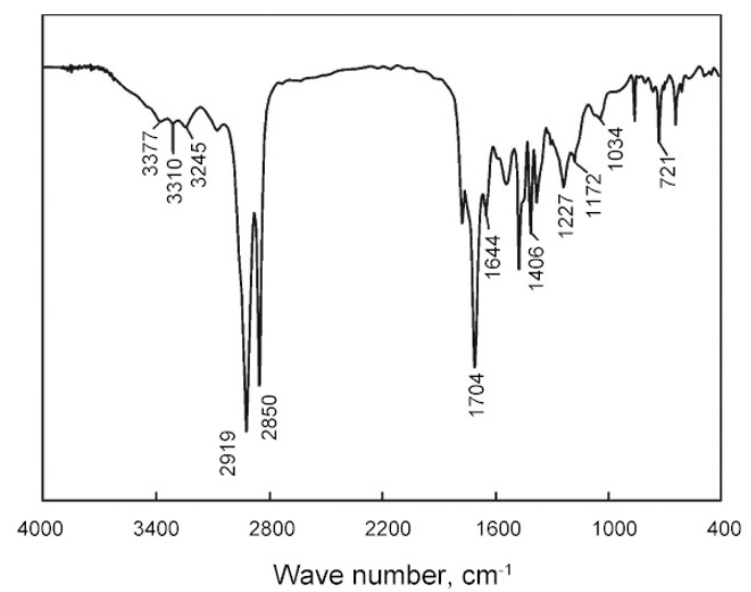

Fig. 2 IR spectra of amidated copolymer MAVA-a

at 2,919 and $2,850 \mathrm{~cm}^{-1}$, the typical swing absorption peaks of characteristic $-\mathrm{CH}_{2}$ - of long-chain alkyl is at $721 \mathrm{~cm}^{-1}$ and the stretching peaks of characteristic $\mathrm{C}=\mathrm{O}$ of maleic anhydride nearly vanishes. The amidation reaction took place as expected. It shows that the stretching peak of characteristic $\mathrm{C}-\mathrm{N}$ is at $1,406 \mathrm{~cm}^{-1}$ and the characteristic of amide group is obvious.

\subsection{Analysis of n-paraffin composition of diesel oil}

No. 0 diesel fuel was examined by gas chromatograph to determine the carbon number distribution of $n$-paraffin and its average carbon number as shown in Fig. 3. By calculation, the average carbon number of the No. 0 fuel from Zhang Jia-Gang was 16.69 , and the content of n-paraffin $\mathrm{C}_{9}$ to $\mathrm{C}_{28}$ accounted for $92 \%$ of the total content.

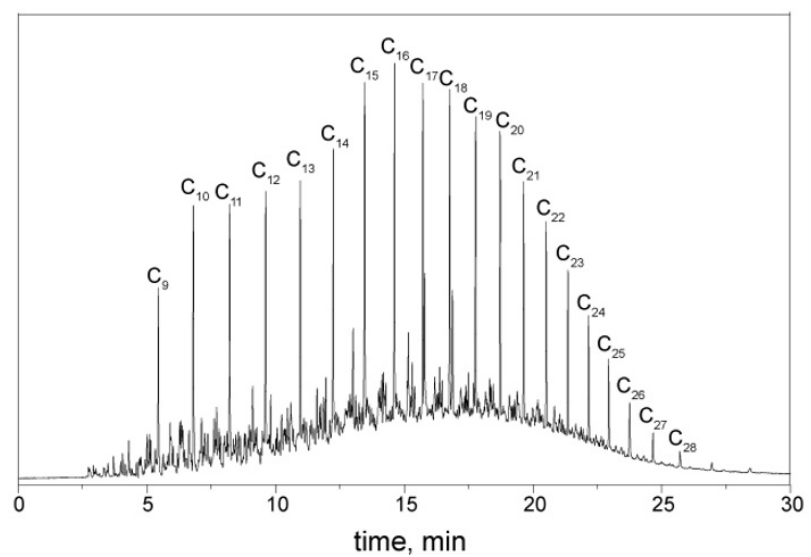

Fig. 3 Carbon number distribution of n-paraffin of No.0 diesel oil from Zhang Jia-Gang

Generally, the susceptibility of diesel fuel to CFI is related to its chemical composition and distillation cut (Guo et al, 1999; Jiang et al, 2002). The susceptibility of diesel fuel to CFI is good when the content of n-paraffin and of the high molecular-weight n-paraffins is low or the content of aromatic hydrocarbons is high. It can be seen from Fig. 3 that there is a normal distribution of carbon number with the high values from $\mathrm{C}_{15}$ to $\mathrm{C}_{19}$ for the No. 0 diesel fuel. The susceptibility of this diesel fuel to CFI is moderate. The interaction of the polymeric additive with the paraffin fraction in the diesel fuel may occur through good matching of the alkyl chain length of the pendant group of the copolymer with that of the paraffin fraction in the diesel fuel (EI-Gamal et al, 1998; Shang et al., 2000a; Shang et al, 2000b). This mode of interaction might be supported (depending on the additive polymeric structure) by the effect of polar nitrogen/oxygen-containing functional groups incorporated in the copolymer that lead to induced dipoles in n-paraffin of the diesel fuel during crystallization. The structure of polymeric improver MAVA-a must be considered in terms of the carbon number of alkyl chains. It is closely related to the average carbon chain length of the paraffin fraction of the diesel fuel and to the polar functional groups incorporated in their structures. Moreover, different diesel fuels have their unique optimal match of alkyl chain length of improvers.

\subsection{Evaluation of the cold flow performance}

According to the Chinese national standard of GB 510-83 (Petroleum products - Determination of solidification point) and SY 2413-83 (Distillation cut fuels-Determination of cold filter plugging point), the cold filter plugging point (CFPP) depression and the solidification point (SP) depression of the diesel fuel from Zhang Jia-Gang were determined at a low temperature on a multi-functional tester (SWXK 402D).

\subsubsection{Comparison between MAVA-a and market available EVA}

The cold flow performance of the CFI was evaluated for No. 0 diesel fuel at an additive concentration of $1000 \mu \mathrm{g} / \mathrm{g}$. The results are listed in Table 1 .

Table 1 Influences of MAVA-a on SP and CFPP of No. 0 diesel fuel

\begin{tabular}{ccccc}
\hline CFI & $\mathrm{CFPP},{ }^{\circ} \mathrm{C}$ & $\triangle \mathrm{CFPP},{ }^{\circ} \mathrm{C}$ & $\mathrm{SP},{ }^{\circ} \mathrm{C}$ & $\triangle \mathrm{SP},{ }^{\circ} \mathrm{C}$ \\
\hline MAVA-a & 0 & -4 & -16 & -16 \\
EVA-1 & 0 & -4 & -20 & -20 \\
EVA-2 & -1 & -5 & -28 & -28 \\
\hline
\end{tabular}

It can be seen that the CFPP of the diesel fuel was lowered by $4{ }^{\circ} \mathrm{C}$ with the improver MAVA-a, by $4{ }^{\circ} \mathrm{C}$ with EVA-1, and by $5{ }^{\circ} \mathrm{C}$ with EVA-2. As a whole, the improver MAVA-a nearly has the same effect as a samples of EVA from two different factories in improving the cold flow performance of the diesel fuel.

3.3.2 Cold flow performance when copolymer MAVA-a used together with EVA

The results are listed in Table 2, where the total additive concentration is $1000 \mu \mathrm{g} / \mathrm{g}$, and the ratio of MAVA-a to EVA is $1: 1$.

Table 2 Influences of mixed DFI on SP and CFPP of diesel fuel

\begin{tabular}{ccccc}
\hline No. & CFPP, ${ }^{\circ} \mathrm{C}$ & $\triangle \mathrm{CFPP},{ }^{\circ} \mathrm{C}$ & $\mathrm{SP},{ }^{\circ} \mathrm{C}$ & $\triangle \mathrm{SP},{ }^{\circ} \mathrm{C}$ \\
\hline With EVA-1 & -2 & -6 & -30 & -30 \\
With EVA-2 & -4 & -8 & -36 & -36 \\
\hline
\end{tabular}


The results given in Table 2 show that the CFPP of the diesel fuel was lowered by $6{ }^{\circ} \mathrm{C}$ and $8{ }^{\circ} \mathrm{C}$ respectively when the improver MAVA-a was used together with EVA-1 and with EVA-2, and the SP was lowered by more than $30^{\circ} \mathrm{C}$.

\subsection{Mechanism of CFPP depression of MAVA-a}

The reactivity ratios of vinyl acetate (VA) and maleic anhydride (MA) at $75^{\circ} \mathrm{C}$ are respectively as follows (Deng and Zhou, 1998; Bray and Merill, 1995): $\gamma_{1}=0.003$; $\gamma_{2}=0.055, \gamma_{1} \gamma_{2}=0.000165$, which belong to the condition of the alternating copolymerization of free radical solution. In this experiment, VA must be dripped into the MA solution to ensure the alternating copolymerization because maleic anhydride has a symmetric non-polar structure, which can not be homopolymerized.

The high dispersing activity of the copolymer may be attributed to the combined effects of the carbonyl oxygen of the maleic anhydride, the polarity exerted by the polar functional groups of the amide and the alkyl chain of the pendant group of the copolymer. The mechanism of CFPP depression can be explained not only by an adsorption mechanism but also by co-crystallization theory (Xue, 2003). The strong point of alternating copolymerization is that the structure of the improver is the symmetrical shape of a herringbone, which is made of different chain lengths of amide chains and ester chains. Accordingly, there are multiple spaces of identical size between the pendant groups. The fact that a long-chain alkyl group can be inserted into the wax crystal in the fuel, and that a polar moiety-amido exists on the surface of the wax crystal results in inhibiting the crystal lattice formation and reducing the wax crystal size (Srivastava et al, 1992).

The performance of the additive can be controlled by proper polymer design. The more similar the polymer structure, i.e. backbone and pendant chain, to the wax components, the better the performance. The interaction may occur through good matching of alkyl chain length of the pendant group of the copolymer with that of the paraffin fraction in the diesel fuel. Therefore, in this experiment $\mathrm{C}_{18}$ was selected as the alkyl chain pendant group to achieve the required performance. The synergetic effect of the compounded improver can promote the polymer to cocrystallize with n-paraffin in the fuel.

\section{Conclusions}

(1) When the dosage of improver MAVA-a was $1000 \mu \mathrm{g} / \mathrm{g}$, the CFPP of the No.0 diesel fuel from Zhang Jia-Gang was lowered by $4{ }^{\circ} \mathrm{C}$, the SP was lowered by $16^{\circ} \mathrm{C}$. At the same total improver concentration, the CFPP was lowered by $6{ }^{\circ} \mathrm{C}$ when the improver MAVA-a was used together with EVA-1, lowered by $8{ }^{\circ} \mathrm{C}$ when used together with EVA-2 and the SP were lowered by more than $30^{\circ} \mathrm{C}$. Thus it can be concluded that the synergetic effect of the compound improver is excellent.

(2) The structure of polymeric improvers could be designed in terms of the carbon number of alkyl chains, which is closely related to the average length of carbon chain and carbon number distribution of n-paraffin fractions in the diesel fuel.

\section{References}

Bray I C and Merill E D. Copolymerization of vinyl acetate with maleic acid. Journal of Polymer Science. 1955. 6 (2): 157-159

Deng X L and Zhou X X. The study of the free-radical solution alternate copolymerization of vinyl acetate with maleic acid. Natural Science Journal of Xiangtan University. 1998. (1): 89-92 (in Chinese)

EI-Gamal I M, Khidr T T and Ghuiba F M. Nitrogen-based copolymers as wax dispersants for paraffinic gas oils. Fuel. 1998. 77 (5): 375-385

Guo C L, Zhang J L and Wang Y P. The progress in research on low temperature flow improvers for diesel fuel. Chemical Industry and Engineering. 1999. 16 (3): 157-162 (in Chinese)

Jiang S H, Zhou J M and Zhang Y Z. Influence of n-alkane on the low temperature behavior of diesel fuel. Chemical Engineering of Oil and Gas. 2002. 31 (5): 243-245 (in Chinese)

Semwal P B and Varshney R G. Predictions of pour, cloud and cold filter plugging point for future diesel fuels with application to diesel blending models. Fuel. 1995. 74 (3): 437-444

Shang H Y, Jiang C Y, Wu M B and Han Z Y. Preparation and evaluation of pour point depressants II. Synergistic effects between components of pour point depressants. Journal of Fuel Chemistry and Technology. 2000a. 28 (3): 225-227 (in Chinese)

Shang H Y, Wang L Q, Jiang S M and Han Z Y. Preparation and evaluation of pour point depressants I. The sensitivity of pour point depressants to diesel. Journal of Fuel Chemistry and Technology. 2000b. 28 (1): 63-66 (in Chinese)

Song Y P, Ren T H, Fu X S and Xu X H. Study on the relationship between the structure and activities of alkyl methacrylate-maleic anhydride polymers as cold flow improvers in diesel fuels. Fuel Processing Technology. 2005. 86: 641-650

Srivastava S P, Tandon R S and Verma, P S. Crystallization behaviour of n-paraffins in Bombay-high middle-distillate wax/gel. Fuel. 1992. 71 (5): 533-537

Xue Z M. Study of diesel fuel low-temperature flow improver AMSV-a. Fine Chemicals. 2003. 20 (3): 182-186 (in Chinese)

Yang J L and Chang J L. Results review for new-type depressant. Oil \& Gas Storage and Transportation. 1997. 16 (5): 5-8, 21 (in Chinese)

Zhang P, Gu X M and Nie G J. Study of the alternating copolymerization of vinyl acetate-maleic anhydride using benzoyl peroxide as initiator. Guangzhou Chemistry. 1995. (1): 22-26 (in Chinese)

(Edited by Zhu Xiuqin) 